\title{
JMR EARLY CAREER SCHOLARS IN MATERIALS SCIENCE ANNUAL ISSUE
}

\section{Introduction}

\author{
Gary L. Messing ${ }^{1}$, Susmita Bose ${ }^{2}$, Mathias Göken ${ }^{3}$, Sarah Morgan ${ }^{4}$ \\ ${ }^{1}$ The Pennsylvania State University, USA, JMR Editor-in-Chief \\ ${ }^{2}$ Washington State University, USA, JMR Associate Editor \\ ${ }^{3}$ University of Erlangen-Nurnberg, Germany, JMR Associate Editor \\ ${ }^{4}$ University of Southern Mississippi, USA, JMR Associate Editor
}

\section{Introduction}

We are pleased to publish the fifth Early Career Scholars in Materials Science annual issue of Journal of Materials Research. The issue highlights young scientists carrying out research in the field of materials. By publishing these papers together, we spotlight this group of early career scholars at a particularly important stage in their careers. Their photos and biographical details are included to personalize the authors and to help other scientists identify them and thus facilitate networking for them. To further extend that exposure, we have published all of these papers on an Open Access basis. It is impressive to see the degree of international representation of the scholars and the efforts taken by this group to carry out state-of-the-art research on a global scale.

The topical coverage represented by the twenty-two papers in this issue completely overlaps with the scope of topics published in $J M R$ and parallels the diversity of subjects represented by the Materials Research Society. Central to all of the papers is a perspective on processing-nanostructureproperty relations - a central theme in materials research. There are four review articles, fifteen invited feature papers and three regular submission papers. A number of papers present innovations in electronic, optical, magnetic, and functional materials. Several papers seek to fabricate materials using novel approaches and material combinations. The power of advanced computation tools is highlighted in two reviews and used in a few other papers. Multiple papers investigate the nanostructure-property relations in ceramics, nano-metals, and polymer composites.

The Early Career Scholars are a diverse group of men and women currently residing in nine countries, and most have spent time at labs and universities outside their home countries. Fifteen of the scholars hold academic positions. We are enthusiastic to see how the careers of this group of young professionals advance in the coming years. We expect they will continue to grow and prosper in the field of materials and assume future leadership roles. Feel free to contact the editors (jmr@mrs.org) if you have any questions about publishing a paper in a future Early Career Scholars in Materials Science annual issue. Enjoy meeting the 2020 Early Career Scholars and reading their outstanding papers! 